\title{
The influence of pre-existing diabetes mellitus on the host immune response and outcome of pneumonia: analysis of two multicentre cohort studies
}

\author{
Sachin Yende, ${ }^{1,2}$ Tom van der Poll, ${ }^{3}$ MinJae Lee, ${ }^{1,4}$ David T Huang, ${ }^{1,2,5}$ \\ Anne B Newman, ${ }^{6}$ Lan Kong, ${ }^{4}$ John A Kellum, ${ }^{1,2}$ Tamara B Harris, ${ }^{7}$ Doug Bauer, \\ Suzanne Satterfield ${ }^{9}$ Derek $C$ Angus, ${ }^{1,2}$ for the GenIMS and Health $A B C$ study
}

\begin{abstract}
- Additional methods are published online only. To view these files please visit the journal online (http://www. thorax.bmj.com).
\end{abstract}

${ }^{1}$ The Clinical Research, Investigation, and Systems Modeling of Acute Illness (CRISMA) Laboratory, University of Pittsburgh, Pittsburgh, Pennsylvania, USA ${ }^{2}$ Department of Critical Care Medicine, University of Pittsburgh, Pittsburgh, Pennsylvania, USA

${ }^{3}$ Center of Infection and Immunity Amsterdam (CINIMA) and Center for Experimental and Molecular Medicine, Academic Medical Center, University of Amsterdam, Amsterdam, The Netherlands

${ }^{4}$ Department of Biostatistics, Graduate School of Public Health, University of Pittsburgh, Pittsburgh, Pennsylvania, USA ${ }^{5}$ Department of Emergency Medicine, University of Pittsburgh, Pennsylvania, USA ${ }^{6}$ Department of Epidemiology, Graduate School of Public Health, University of Pittsburgh, Pittsburgh, Pennsylvania, USA ${ }^{7}$ Laboratory of Epidemiology, Demography and Biometry, National Institute on Ageing Bethesda, Maryland, USA

${ }^{8}$ Department of Epidemiology and Biostatistics and Medicine, University of California, San Francisco, California, USA ${ }^{9}$ Department of Preventive Medicine, University of Tennessee, Memphis, Tennessee, USA

\section{Correspondence to} Sachin Yende, CRISMA laboratory, Department of Critical Care Medicine, University of Pittsburgh, 642A Scaife Hall, 3550 Terrace Street, Pittsburgh, PA 15261, USA; yendes@upmc.edu

Received 11 February 2010 Accepted 28 June 2010

\begin{abstract}
Background Although diabetes mellitus is implicated in susceptibility to infection, the association of diabetes with the subsequent course and outcome is unclear.

Methods A retrospective analysis of two multicentre cohorts was carried out. The effect of pre-existing diabetes on the host immune response, acute organ function and mortality in patients hospitalised with community-acquired pneumonia (CAP) in the GenIMS study ( $n=1895$ ) and on mortality following either CAP or non-infectious hospitalisations in the population-based cohort study, Health ABC $(n=1639)$ was determined. Measurements included the mortality rate within the first year, risk of organ dysfunction, and immune responses, including circulating inflammatory (tumour necrosis factor, interleukin 6, interleukin 10), coagulation (Factor IX, thrombin-antithrombin complexes, antithrombin), fibrinolysis (plasminogen-activator inhibitor-1 and D-dimer) and cell surface markers (CD120a, CD120b, human leucocyte antigen (HLA)-DR, Toll-like receptor-2 and Toll-like receptor-4).
\end{abstract}

Results In GenIMS, diabetes increased the mortality rate within the first year after CAP (unadjusted HR 1.41, $95 \% \mathrm{Cl} 1.12$ to $1.76, p=0.002$ ), even after adjusting for pre-existing cardiovascular and renal disease (adjusted HR 1.3, 95\% Cl 1.03 to 1.65, $p=0.02$ ). In Health $A B C$, diabetes increased the mortality rate within the first year following CAP hospitalisation, but not after hospitalisation for non-infectious illnesses (significant interaction for diabetes and reason for hospitalisation $(p=0.04)$; HR for diabetes on mortality over the first year after CAP $1.87,95 \% \mathrm{Cl} 0.76$ to $4.6, \mathrm{p}=0.16$, and after non-infectious hospitalisation $1.16,95 \% \mathrm{Cl} 0.8$ to 1.6 , $p=0.37$ ). In GenIMS, immediate immune response was similar, as evidenced by similar circulating immune marker levels, in the emergency department and during the first week. Those with diabetes had a higher risk of acute kidney injury during hospitalisation $339.3 \%$ vs $31.7 \%, p=0.005$ ) and they were more likely to die due to cardiovascular and kidney disease (34.4\% vs $26.8 \%$ and $10.4 \%$ vs $4.5 \%, p=0.03$.

Conclusions Pre-existing diabetes was associated with a higher risk of death following CAP. The mechanism is not due to an altered immune response, at least as measured by a broad panel of circulating and cell surface markers, but may be due to worsening of pre-existing cardiovascular and kidney disease.

It is a long-standing medical axiom that diabetes mellitus is a risk factor for infection. ${ }^{1}{ }^{2}$ However, once infection occurs, the effect of diabetes on mortality is less clear. Some studies suggest that diabetes is associated with higher mortality after an infection, but others show no association. ${ }^{3-7}$ Previous studies that showed the association between diabetes and higher mortality may be confounded by higher prevalence of pre-existing chronic conditions, such as chronic kidney disease (CKD) or cardiovascular disease. ${ }^{8-10}$

If diabetes indeed is associated with higher mortality after infection, underlying mechanisms are unknown. Several mechanisms could explain these survival differences. For instance, animal and human models of infection suggest that immune abnormalities in diabetes, such as higher proinflammatory ${ }^{11} 12$ procoagulant and antifibrinolytic activity, ${ }^{13}{ }^{14}$ and higher expression of pathogen recognition cell surface receptors, ${ }^{15}{ }^{16}$ could worsen during acute illness and increase mortality. An alternative mechanism for higher mortality among those with diabetes is increased risk of acute organ dysfunction due to higher chronic disease burden.

We therefore examined two multicentre observational cohort studies to understand the effect of diabetes on the host immune response and outcomes of pneumonia. We analysed a cohort of patients with community-acquired pneumonia (CAP) enrolled in the Genetic and Inflammatory Markers of Sepsis (GenIMS) study, and the subgroup of the population-based cohort, Health, Ageing, and Body Composition (Health $A B C$ ) study, who required hospitalisation. In both cohorts, we tested the hypothesis that pre-existing diabetes is associated with increased mortality within the first year, independent of pre-existing chronic diseases. We then determined whether higher mortality is attributable to CAP per se by comparing survival differences over the first year in Health $\mathrm{ABC}$ between $\mathrm{CAP}$ and non-infectious hospitalisations. Finally, in GenIMS we tested the hypothesis that survival differences between those with and without diabetes were due to differences in immune response and a higher risk of acute organ dysfunction during infection.

\section{METHODS}

\section{Subjects and design}

We analysed subjects enrolled in the GenIMS cohort to assess differences in mortality, organ dysfunction and immune response. GenIMS is a prospective multicentre observational cohort of 
subjects with CAP enrolled in emergency departments (EDs) of 28 academic and community hospitals in four US regions - that is, southwestern Pennsylvania, Connecticut, southern Michigan and western Tennessee. Eligibility criteria are shown in table 1. Of the 2320 subjects enrolled, we excluded 288 patients because they were discharged from the ED and an additional 137 patients because the clinical team ruled out CAP during the first 3 days of hospitalisation, thus restricting the analysis to the remaining 1895 subjects.

To assess whether higher mortality is attributable to CAP, we analysed subjects enrolled in the Health ABC study, a population-based observational cohort of 70- to 79-year-old well-functioning participants. Health ABC participants were enrolled from the same geographic regions as GenIMS, including southwestern Pennsylvania (Pittsburgh) and western Tennessee (Memphis). Of the 3075 enrolled, we analysed 1645 (53.5\%) subjects who were hospitalised at least once during the first 5 years of follow-up. We excluded an additional six subjects whose diabetes status was not known, restricting the analysis to the remaining 1639 subjects. Methods to ascertain hospitalisation have been described previously. ${ }^{20}$ Briefly, we assessed outcomes after the first major hospitalisation following enrolment, comparing mortality rate within the first year among those initially hospitalised for CAP and non-infectious illnesses (see appendix 1). For each cohort, the Institutional Review Boards at each site approved the study. Informed consent was obtained from participants or next of kin for GenIMS and from participants for Health ABC.

\section{Outcome and clinical variables}

For GenIMS and Health ABC cohorts, the primary outcome variable was all-cause mortality rate within the first year (table 1). We examined several additional secondary outcome variables in
GenIMS. We examined cause-specific mortality using National Death Index codes. The validity of this method has been described previously. ${ }^{21} 22$ To compare risk of developing acute organ dysfunction during hospitalisation, we compared risk of severe sepsis (infection plus organ dysfunction) using Consensus criteria, ${ }^{17}$ and the risk of individual organ dysfunction for six organ systems. For acute kidney injury, we used the RIFLE criteria. ${ }^{23}$ The RIFLE criteria classifies acute kidney injury into three categories of severity using changes in serum creatinine and urine output (Risk, Injury, and Failure corresponding to mild, moderate, and severe kidney injury, respectively). Finally, we used measures of severity of illness at hospital presentation, including APACHE III (Acute Physiology and Chronic Health Evaluation III) and the Pneumonia Severity Index (PSI). ${ }^{24} 25$ Details of methods to assess co-morbid conditions in both cohorts, including diabetes, are included in table 1. Microbiological characteristics were available only in GenIMS and were assessed using blood and sputum cultures (see Online repository).

\section{Laboratory procedures}

We assessed differences in the immune responses to infection in GenIMS during hospitalisation between those with and without diabetes by comparing changes in circulating concentrations of biomarkers within inflammatory (tumour necrosis factor $\alpha(\mathrm{TNF} \alpha)$, interleukin 6 (IL-6), IL-10), coagulation (Factor IX, thrombin-antithrombin complexes (TATs), antithrombin) and fibrinolysis (plasminogen activator inhibitor-1 (PAI-1) and D-dimer) systems, and expression of cell surface markers (CD120a and CD120b (signalling receptors for TNF), human leucocyte antigen (HLA)-DR and Toll-like receptor2 (TLR2) and TLR4) on presentation to the ED and over the first week. Details of sample collection and processing have been described previously. ${ }^{26}$

Table 1 Characteristics, eligibility criteria and methods to determine clinical and outcome measures in Genetic and Inflammatory Markers of Sepsis (GenIMS) and Health, Ageing and Body Composition (Health $A B C$ ) cohorts

\begin{tabular}{|c|c|c|}
\hline Variable & GenIMS study & Health ABC study \\
\hline
\end{tabular}

Exclusion criteria

Enrolment period

Ascertainment of outcome measures All-cause mortality

\section{community-acquired pneumonia}

Transfer from another hospital, discharge from an acute care hospital within the previous 10 days, diagnosis of pneumonia within the previous 30 days, chronic dependency on mechanical ventilation, cystic fibrosis, active pulmonary tuberculosis, admission for palliative care, prior enrolment in the study, incarceration or pregnancy

$$
\text { 2001-2003 }
$$

By study nurses during hospitalisation and by National Death Index after hospital discharge

National Death Index codes

Defined as pneumonia and organ dysfunction, based on the International Consensus Criteria ${ }^{17}$

Severe sepsis

Co-morbid conditions

Diabetes mellitus

Cardiovascular disease

CKD
Self-report and review of medications (use of oral hypoglycaemic medications or insulin)

Self-report and review of medications

Self-report and prehospitalisation creatinine measurements, if available. We also used creatinine measurements available during hospitalisation to exclude CKD 
We used an automated chemiluminescent immunoassay analyser (IMMULITE, Diagnostic Products, Los Angeles, California, USA) to analyse TNF, IL-6 and IL-10. We analysed coagulation and fibrinolysis markers in a random subset of 734 subjects by a commercial laboratory (Esoterix, Agoura Hills, California, USA). Specific methods and kits used were: D-dimer, latex immunoassay (Diagnostica Stago, Parsippany, New Jersey, USA); PAI-1, bio immunoassay (Biopool Chromolize, Biopool International, Ventura, California, USA); antithrombin, chromogenic (BioMerieux, Rhône-Alpes, France); Factor IX, clot (BioMerieux); and TAT, ELISA (Behring, King of Prussia, Pennsylvania, USA).

We analysed cell surface markers on ED presentation and on the third and seventh day. We analysed cell surface markers in a subset of 624 subjects enrolled in hospitals located within 60 miles of the University of Pittsburgh because samples for cell surface markers have to be analysed within $48 \mathrm{~h}$. We obtained fluorochrome- or biotin-conjugated antibodies from eBioScience (San Diego, California, USA) for TLR2, TLR4 and HLA-DR, and from Invitrogen (Carlsbad, California, USA) for CD120a and CD120b. We incubated these antibodies with whole blood, lysed the red blood cells and then washed, fixed and stored the remaining cells at $-4^{\circ} \mathrm{C}$. We acquired cell surface marker data within $48 \mathrm{~h}$ of fixation on a BD FACSVantage SE flow cytometer (San Jose, California, USA) and used BD CellQuest software. Additional details of the assays for all markers are included in the Online repository.

\section{Statistical analyses}

We conducted univariate comparisons of clinical characteristics for subjects with and without diabetes using $\chi^{2}$ and Student $t$ test and their non-parametric counterparts when necessary in both cohorts. We compared the mortality rate within the first year by constructing Kaplan-Meier survival curves for subjects with and without diabetes and used the Cox proportional hazards model. For the proportional hazards model, we confirmed that the HRs were similar over different intervals. We constructed serial models, including unadjusted, adjusted for demographic characteristics (age, sex and race), and adjusted for demographic characteristics and chronic diseases associated with diabetes, such as cardiovascular disease and CKD. To assess if mortality differences were attributable to CAP, we compared mortality in those hospitalised initially for CAP and other noninfectious illnesses in Health ABC. We tested for an interaction between diabetes and the reason for initial hospitalisation and mortality rate within the first year.

We assessed mechanisms of increased mortality in GenIMS. We compared risk of severe sepsis and individual organ dysfunction using logistic regression analyses. We compared differences in immune response to infection, comparing biomarkers and cell surface markers in subjects with and without diabetes at ED presentation and during the first week of hospital stay. We used regression analysis with mixed models to account for correlation of these markers over time. We also compared inflammatory and coagulation biomarkers at hospital discharge in the subset that was discharged alive and appeared to have recovered clinically to compare resolution of the immune response. We have previously shown that those with an unresolved immune response, as evidenced by higher concentrations of biomarkers at hospital discharge, had higher mortality over the first year after hospital discharge. ${ }^{27}$ To account for biomarkers that were truncated because they were below the detection thresholds, we used Tobit models to compare biomarker concentrations. ${ }^{28}$ Due to the large number of comparisons for the cell surface markers, we reported $p$ values adjusted for false discovery rate at $0.05 .^{29}$

\section{RESULTS \\ Baseline characteristics of the GenIMS cohort}

Table 2 shows pre-hospitalisation characteristics for 1895 subjects enrolled in GenIMS. The average age of the cohort was 67 years and two-thirds had a history of smoking. Respiratory disease was the most common chronic disease and occurred in approximately a third of the cohort, and cardiovascular disease occurred in a quarter of the cohort. Diabetes occurred in 384 $(20.3 \%)$ subjects. The prevalence of other chronic diseases, such as $\mathrm{CKD}$, cancer and HIV, was low $(<5 \%)$.

Subjects with diabetes, on average, were 3 years older than subjects without diabetes, but results did not reach statistical significance. Those with diabetes were more likely to have CKD $(10.4 \%$ vs $3.4 \%, p<0.0001)$ and cardiovascular disease $(42.9 \%$ vs $21.3 \%, \mathrm{p}<0.0001)$. HIV was more prevalent among those without diabetes $(2.4 \%$ vs $0.3 \%, p=0.02)$ and only one subject with

Table 2 Demographic and clinical characteristics of all subjects, stratified by diabetes, enrolled in the Genetic and Inflammatory Markers of Sepsis (GenIMS) and Health, Ageing and Body Composition (Health $\mathrm{ABC}$ ) study

\begin{tabular}{|c|c|c|c|c|c|c|}
\hline \multirow[b]{2}{*}{ Variable } & \multicolumn{3}{|c|}{ GenIMS study $(n=1895)$} & \multicolumn{3}{|c|}{ Health ABC study (n=1645) } \\
\hline & All subjects & $\begin{array}{l}\text { With diabetes } \\
(\mathrm{n}=384)\end{array}$ & $\begin{array}{l}\text { Without diabetes } \\
(n=1511)\end{array}$ & All subjects & $\begin{array}{l}\text { With diabetes } \\
(n=299)\end{array}$ & $\begin{array}{l}\text { Without diabetes } \\
(n=1340)\end{array}$ \\
\hline \multicolumn{7}{|l|}{ Demographics } \\
\hline Age, mean (SD, median) & $67.2(16.8,72)$ & $69.3(14.5,73)$ & $66.7(17.3,71)$ & $73.7(2.8,74)$ & $73.7(2.8,73)$ & $73.7(2.8,74)$ \\
\hline Sex, female, $\mathrm{n}(\%)$ & $910(48.0)$ & $177(46.1)$ & $733(48.5)$ & $766(46.7)$ & $132(44.1)$ & $634(47.3)$ \\
\hline Race, white, n (\%) & $1529(80.7)$ & $307(80)$ & $1222(80.1)$ & $939(57.2)$ & $127(42.4)$ & $812(60.6)$ \\
\hline \multicolumn{7}{|l|}{ Health behaviours } \\
\hline Ever smoked, n (\%) & $1262(66.6)$ & $244(63.5)$ & $1018(67.4)$ & $997(60.9)$ & $183(61.6)$ & $814(60.8)$ \\
\hline \multicolumn{7}{|l|}{ Chronic health conditions } \\
\hline Respiratory disease, $\mathrm{n}(\%)$ & $718(37.9)$ & $148(38.5)$ & $570(37.7)$ & $320(24.2)$ & $64(27.4)$ & $256(19.3)$ \\
\hline Cardiovascular disease, $\mathrm{n}(\%)$ & $488(25.7)$ & $165(42.9)$ & $323(21.3)^{*}$ & $438(27.3)$ & $97(35.9)$ & $341(25.9)^{*}$ \\
\hline CKD, n (\%) & $92(4.8) \dagger$ & $40(10.4)$ & $52(3.4)^{*}$ & $403(24.5)$ & $102(34.1)$ & $301(22.4)^{*}$ \\
\hline Cancerf, n (\%) & $94(5.0)$ & $22(5.7)$ & $72(4.8)$ & - & - & - \\
\hline HIV $\ddagger, n(\%)$ & $37(2.0)$ & $1(0.3)$ & $36(2.4)^{*}$ & - & - & - \\
\hline
\end{tabular}

${ }^{*} \mathrm{p}<0.05$ for comparison among subjects with and without diabetes

†CKD diagnosis was available in $1571(82.9 \%)$ subjects in GenIMS. In the remaining 324 subjects, CKD could not be ruled out based on history and because their plasma creatinine values were elevated on the first day of hospitalisation for pneumonia.

$\ddagger$ Health $\mathrm{ABC}$ excluded subjects with active cancer and none reported HIV.

CKD, chronic kidney disease. 
diabetes had HIV. No differences were seen in the prevalence of smoking, cancer and respiratory disease between the two groups.

Blood or sputum cultures to determine microbiological aetiology were obtained in 1606 (84.7\%) subjects within 48 h of ED presentation. An aetiological agent was identified in 186 (11.5\%) subjects, and this frequency was similar among those with and without diabetes $(12.6 \%$ vs $11.3 \%, \mathrm{p}=0.5)$. Among subjects in whom an aetiological agent was identified, Gram-negative organisms were more common among those with diabetes, Gram-positive organisms were more common among those without, and no differences were seen in the frequency of mixed or anaerobic organisms (7.5\% vs $3.1 \%, 4.2 \%$ vs $7.5 \%$, and $1 \%$ vs $0.7 \%$ for Gram-negative, Gram-positive and mixed or anaerobic organisms, respectively, $\mathrm{p}=0.001)$.

\section{Diabetes was associated with higher acute organ dysfunction in GenIMS}

At ED presentation, subjects with diabetes had greater illness severity, as evidenced by higher PSI scores (111.1 vs 96.9, $p<0.0001)$, higher frequency of subjects with diabetes in PSI classes IV and V $(68.7 \%$ vs $53.6 \%, p<0.0001)$, and higher APACHE III scores (59.5 vs 55.2, $\mathrm{p}<0.0001)$. When points for the physiology components of the APACHE III score were compared, those with diabetes had a higher score compared with those without diabetes (42.6 vs 39.8, p<0.0001).

Subjects with diabetes had higher risk of severe sepsis compared with those without diabetes $(34.6 \%$ vs $29.7 \%$, OR $1.25,95 \%$ CI 0.99 to 1.58 ), but results were not statistically significant $(p=0.06)$. This association was not statistically significant when adjusted for age, sex, race, and pre-existing cardiovascular disease and CKD (OR 1.23, 95\% CI 0.96 to 1.59, $\mathrm{p}=0.09)$.

Diabetes increased the risk of acute kidney injury (30.2\% vs $22.9 \%$ for subjects with and without diabetes, $\mathrm{p}=0.007$ ) and this association remained significant when adjusted for age, sex and race $(p=0.02)$. The association persisted when subjects with CKD were excluded (29.3\% vs $23.6 \%, p=0.05)$. The higher risk of acute kidney injury was mainly due to increased risk of moderate (RIFLE-I) kidney injury (7.6\% vs $3.6 \%$ ), and only small differences were observed in the risk of mild (RIFLE-R) $(10.9 \%$ vs $9.6 \%)$ and severe kidney injury (RIFLE-F) (11.6\% vs $9.6 \%)$. No differences were observed in the risk of respiratory, neurological, cardiovascular, coagulation and liver dysfunction (table 3). Furthermore, those with and without diabetes were equally likely to require mechanical ventilation $(6.8 \%$ vs $7 \%, p=0.87)$ and intensive care unit (ICU) admission (14.1\% vs $16.5 \%$, $\mathrm{p}=0.25$ ), and only a small difference was seen in the median length of hospital stay (7.8 vs 7.4 days, $\mathrm{p}=0.02$ ).

\section{Diabetes was associated with higher mortality in GenIMS}

Subjects with diabetes had higher mortality rates over the first year (unadjusted HR was $1.41,95 \%$ CI 1.12 to $1.76, p=0.002$; figure 1 and table 4). The $\mathrm{HR}$ remained unchanged and the association remained statistically significant when adjusted for demographics (HR 1.32, 95\% CI 1.05 to $1.66, p=0.01$ ) and additionally adjusting for higher burden of cardiovascular disease and CKD (HR 1.3, 95\% CI 1.03 to 1.65, $\mathrm{p}=0.02$ ).

The mortality at 1 year in individuals who developed acute kidney injury was higher compared with those who did not develop it, in both subjects with and without diabetes, but results did not reach statistical significance among subjects with diabetes $(30.7 \%$ vs $21.4 \%, p=0.08$ and $28.7 \%$ vs $14.3 \%, p<0.0001$ in subjects with and without acute kidney injury stratified by presence and absence of diabetes, respectively).
Table 3 Risk of severe sepsis and organ dysfunction among subjects with and without diabetes*

\begin{tabular}{lccl}
\hline Variable & With diabetes & Without diabetes & p Value \\
\hline $\begin{array}{l}\text { Risk of severe sepsis, } \mathrm{n}(\%) \\
\text { Organ dysfunction, } \mathrm{n}(\%)\end{array}$ & $133(34.6)$ & $449(29.7)$ & 0.06 \\
$\quad$ & $76(19.8)$ & $254(16.8)$ & 0.17 \\
Respiratory & $15(3.9)$ & $60(4.0)$ & 0.95 \\
Cardiovascular & $116(30.2)$ & $346(22.9)$ & 0.007 \\
Renal & $1(0.3)$ & $11(0.7)$ & 0.27 \\
Liver & $31(8.1)$ & $83(5.5)$ & 0.06 \\
Neurological & $4(1.0)$ & $19(1.3)$ & 0.49 \\
Coagulation & &
\end{tabular}

*Consensus criteria were used to define severe sepsis and acute organ dysfunction, except for renal dysfunction where the RIFLE criteria were used. ${ }^{17}{ }^{23}$

Causes of death were different in those with and without diabetes $(p=0.03)$. Deaths due to cardiovascular disease and kidney disease were higher among those with diabetes $34.4 \%$ vs $26.8 \%$ and $10.4 \%$ vs $4.5 \%$ for cardiovascular and kidney disease, respectively). Deaths due to cancer were lower among those with diabetes $(12.5 \%$ vs $26.5 \%)$, but no differences were observed in deaths due to infection, chronic respiratory disease and other causes $(15.6 \%$ vs $15 \%, 16.7 \%$ vs $14.7 \%$ and $10.4 \%$ vs $12.5 \%$ among those with and without diabetes for infection, chronic respiratory disease and other causes, respectively).

\section{Diabetes was associated with higher mortality after pneumonia} compared with non-infectious illnesses in Health ABC

The clinical characteristics of subjects in Health $A B C$ at enrolment are shown in table 2 . The average age was 73 years and approximately half were whites and females. The prevalence of diabetes was similar in Health ABC and GenIMS ( $n=299,18.2 \%$ and $n=384,20.3 \%$ in Health $A B C$ and GenIMS cohorts, respectively). Compared with GenIMS, the prevalence of respiratory disease was lower in Health $\mathrm{ABC}(24.2 \%$ vs $37.9 \%)$, whereas CKD was more common in Health $\mathrm{ABC}(24.5 \%$ vs 9.8\%). Similar to GenIMS, subjects with diabetes enrolled in Health $\mathrm{ABC}$ had a higher prevalence of CKD $(34.1 \%$ vs $22.4 \%$, $\mathrm{p}<0.0001)$ and cardiovascular disease $(35.9 \%$ vs $25.9 \%, \mathrm{p}=0.03)$.

An interaction was observed between diabetes and the reason for the initial hospitalisation and mortality within the first year. In the unadjusted model, pre-existing diabetes was associated with higher risk of death after hospitalisation for CAP compared with those hospitalised for non-infectious illnesses (HR 1.87, $95 \%$ CI 0.76 to 4.6 , and $1.16,95 \%$ CI 0.8 to 1.6 for those hospitalised for CAP and non-infectious illnesses, respectively; interaction $\mathrm{p}=0.04$ (table 4)). This interaction persisted in models adjusted for demographics (HR 1.82, 95\% CI 0.72 to 4.62, and $1.08,95 \%$ CI 0.76 to 1.52 for those hospitalised for

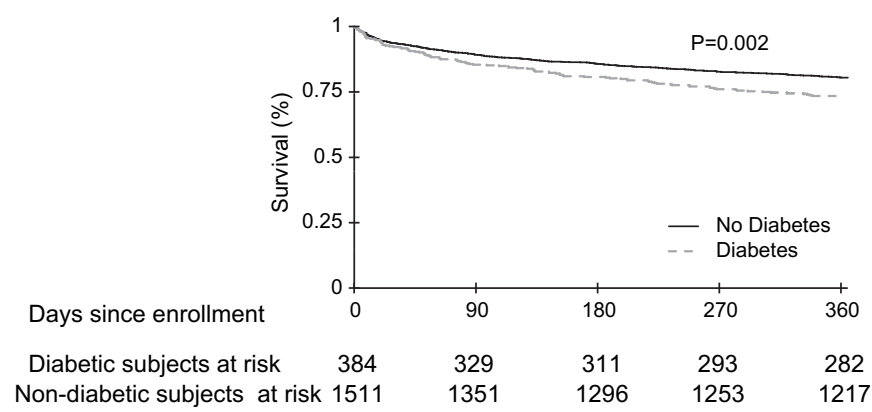

Figure 1 Kaplan-Meier survival curves over 1 year showing higher risk of death for subjects with diabetes compared with those without diabetes following hospitalisation for community-acquired pneumonia in GenIMS. 
Table 4 Unadjusted and adjusted HRs with 95\% Cls for mortality over 1 year for subjects with diabetes compared with those without using Cox proportional hazards model

\begin{tabular}{|c|c|c|c|c|c|c|c|}
\hline \multirow[b]{3}{*}{ Variables } & \multicolumn{2}{|l|}{ GenIMS study } & \multicolumn{5}{|l|}{ Health ABC study } \\
\hline & \multicolumn{2}{|c|}{ Pneumonia hospitalisation } & \multicolumn{2}{|c|}{ Pneumonia hospitalisation } & \multicolumn{2}{|c|}{$\begin{array}{l}\text { Hospitalisation for } \\
\text { non-infectious illness }\end{array}$} & \multirow{2}{*}{$\begin{array}{l}\text { Interaction } \\
\text { p value* }\end{array}$} \\
\hline & HR with 95\% CI & $\overline{p \text { Value }}$ & HR with 95\% Cl & $\overline{p \text { Value }}$ & HR with 95\% Cl & $\overline{p \text { Value }}$ & \\
\hline Unadjusted & 1.41 (1.12 to 1.76$)$ & 0.002 & $1.87(0.76$ to 4.6$)$ & 0.16 & $1.16(0.8$ to 1.6$)$ & 0.37 & 0.04 \\
\hline Adjusted for age, race, sex & $1.32(1.05$ to 1.66$)$ & 0.01 & $1.82(0.72$ to 4.62$)$ & 0.2 & $1.08(0.76$ to 1.52$)$ & 0.65 & 0.04 \\
\hline $\begin{array}{l}\text { Adjusted for age, race, sex, } \\
\text { cardiovascular and kidney disease }\end{array}$ & $1.3(1.03$ to 1.65$)$ & 0.02 & 1.8 (0.66 to 4.92$)$ & 0.25 & $1.06(0.75$ to 1.51$)$ & 0.7 & 0.09 \\
\hline
\end{tabular}

${ }^{*} p$ Value for interaction between diabetes and the reason for initial hospitalisation (pneumonia and non-infectious illness) on mortality over 1 year.

CAP and non-infectious illnesses, respectively; interaction $\mathrm{p}=0.04$ ) and additionally adjusted for pre-existing cardiovascular disease and CKD (HR 1.8, 95\% CI 0.66 to 4.92 , and 1.06, 95\% CI 0.75 to 1.51 for those hospitalised for CAP and non-infectious illnesses, respecteively; interaction $\mathrm{p}=0.09$ ). Forty-one participants who were hospitalised initially for non-infectious illnesses were subsequently hospitalised for infections. Excluding these subjects did not alter the results, and an interaction between diabetes, reason for hospitalisation and mortality within the first year persisted (unadjusted HR 1.87, 95\% CI 0.76 to 4.60 , and $0.97,95 \%$ CI 0.66 to 1.41 for those hospitalised for CAP and noninfectious illnesses, respectively; interaction $\mathrm{p}=0.01$ ).

\section{Diabetes did not alter immune response after CAP hospitalisation}

In GenIMS, the circulating concentrations of inflammatory (TNF, IL-6 and IL-10), coagulation (antithrombin, Factor IX and TAT complexes) and fibrinolysis (PAI-1 and D-dimer) biomarkers were similar among subjects with and without diabetes at ED presentation and over the first week of hospitalisation (figure 2). No differences were seen in expression of CD120a, CD120b, HLA-DR, TLR4 and TLR2 on monocytes between those with and without diabetes (figure 3). Expression of TLR2 on granulocytes was higher among subjects with diabetes on day 1 and 3 , but higher among those without diabetes on day $7(p=0.04)$.
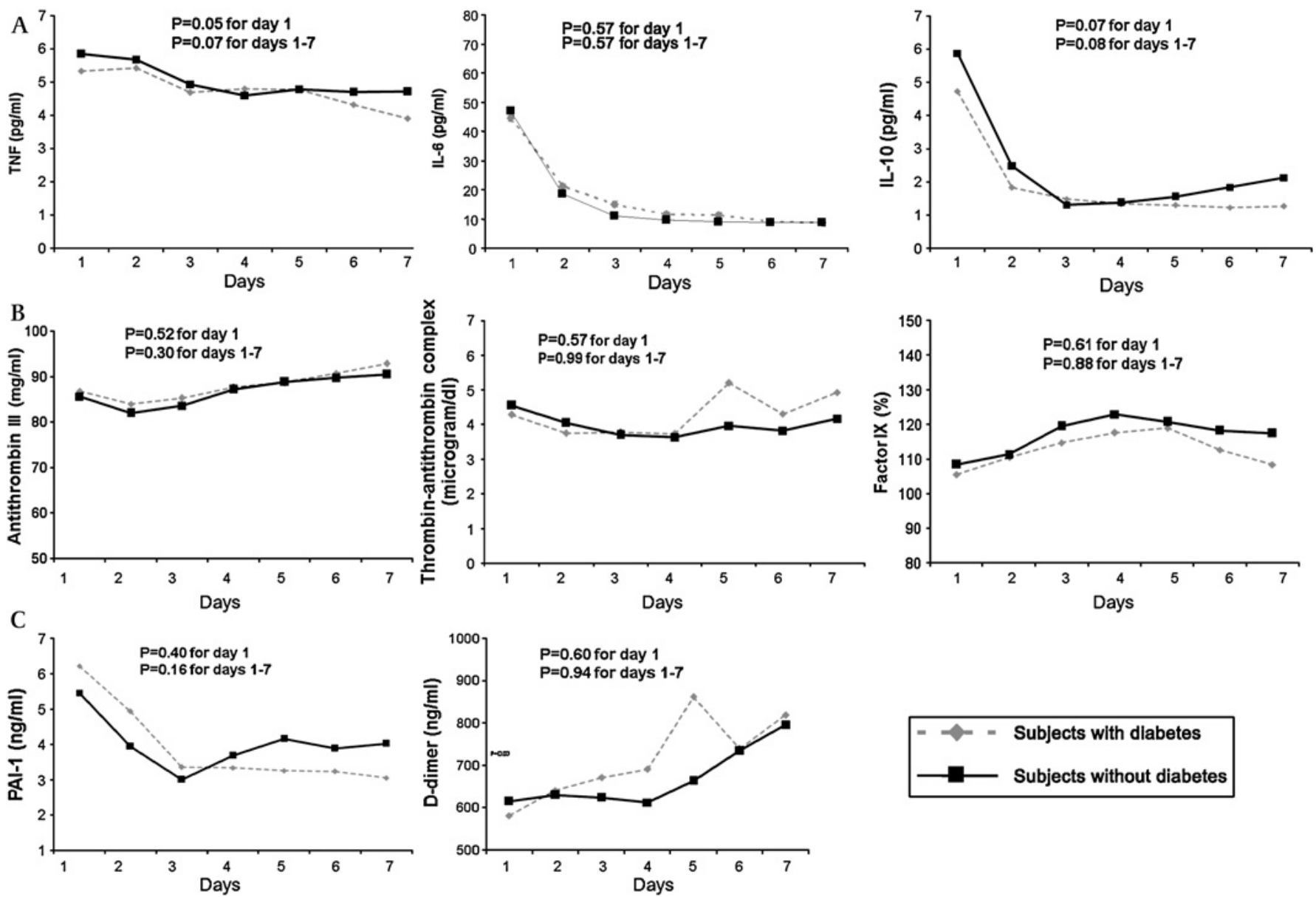

Figure 2 No differences were observed in geometric means of inflammatory (tumour necrosis factor, interleukin 6 (IL-6) and IL-10 shown in A), coagulation (Factor IX, antithrombin and thrombin-antithrombin complexes shown in B) and fibrinolysis (plasminogen activator-1 PAI-1) and D-dimer shown in C) biomarkers in 1427, 734 and 734 subjects with and without diabetes, respectively. Biomarkers were measured on presentation to the emergency department and over the first week of hospitalisation, and $p$ values are shown for these comparisons. 
At hospital discharge, those with diabetes had higher IL-6 concentrations compared with those without, but these differences were small (8.67 vs $7.41 \mathrm{pg} / \mathrm{ml}, \mathrm{p}=0.03$ ) (table 5). No differences were observed in other biomarkers at hospital discharge.

\section{Plasma glucose concentrations}

Plasma glucose was available at ED presentation in most GenIMS subjects $(n=1795,94.7 \%)$. Those with diabetes had higher median plasma glucose compared with those without diabetes $(190 \mathrm{mg} / \mathrm{dl}$, IOR 136-256 mg/dl vs $117 \mathrm{mg} / \mathrm{dl}$, IOR 99-143 mg/dl, $\mathrm{p}<0.0001)$.

Regardless of diabetes, non-survivors at 1 year had higher median blood glucose on ED presentation compared with survivors, but differences were small $(130 \mathrm{mg} / \mathrm{dl}$, IOR $103-177 \mathrm{mg} / \mathrm{dl}$ vs $122 \mathrm{mg} / \mathrm{dl}$, IOR $102-158 \mathrm{mg} / \mathrm{dl}, \mathrm{p}=0.02)$. Hyperglycaemia at ED presentation (glucose $>200 \mathrm{mg} / \mathrm{dl}$ ) was associated with higher mortality within the first year (HR 1.31, $95 \%$ CI 1.01 to $1.69, \mathrm{p}=0.03$ ). However, results did not reach statistical significance when adjusted for age, sex and race (HR $1.27,95 \%$ CI 0.98 to $1.64, \mathrm{p}=0.06$ ). Furthermore, the HRs were different when the analyses were stratified by presence or absence of diabetes (HR $0.89,95 \%$ CI 0.6 to $1.32, p=0.57$ in subjects with diabetes and HR $1.4,95 \%$ CI 0.98 to $2.11, p=0.06$ in subjects without diabetes).

\section{DISCUSSION}

We found that diabetes was common and present in $20 \%$ of subjects with CAP. Compared with subjects without diabetes, those with diabetes had a higher mortality within the first year after CAP and a quarter died at 1 year. The higher mortality was not confounded by higher burden of pre-existing cardiovascular disease and CKD. Furthermore, diabetes was associated with higher mortality following hospitalisation for CAP compared with hospitalisation for non-infectious illnesses. These results suggest that higher mortality within the first year among individuals with diabetes is attributable to the pneumonia hospitalisation. Diabetes did not modify the immune response in a broad panel of circulating inflammatory, coagulation, fibrinolysis and cell surface markers, suggesting that differences in immune response are unlikely to explain survival differences. Based on patterns of organ dysfunction observed during the hospital course and cause-specific mortality, the higher incidence of acute kidney injury and acceleration of underlying cardiovascular disease may mediate higher mortality within the first year after CAP among subjects with diabetes.

Our results showing survival differences due to diabetes after CAP hospitalisation have several strengths. First, the higher hazards of death due to diabetes were similar in two cohorts, although results did not reach statistical significance in Health $\mathrm{ABC}$ due to the small number of CAP events. Secondly, we
Figure 3 No differences were observed in cell surface markers on presentation to the emergency department and on the third and seventh day in 624 subjects with and without diabetes. Values for cell surface markers were reported as the mean channel fluorescence of cells positive for a given cell surface marker. $p$ Values are shown for comparisons over time. Only expression of Toll receptor 2 (TLR2) on granulocytes appeared to be higher among subjects with diabetes on day 1 and 3 and were higher in those without diabetes on day $7(p=0.04)$.
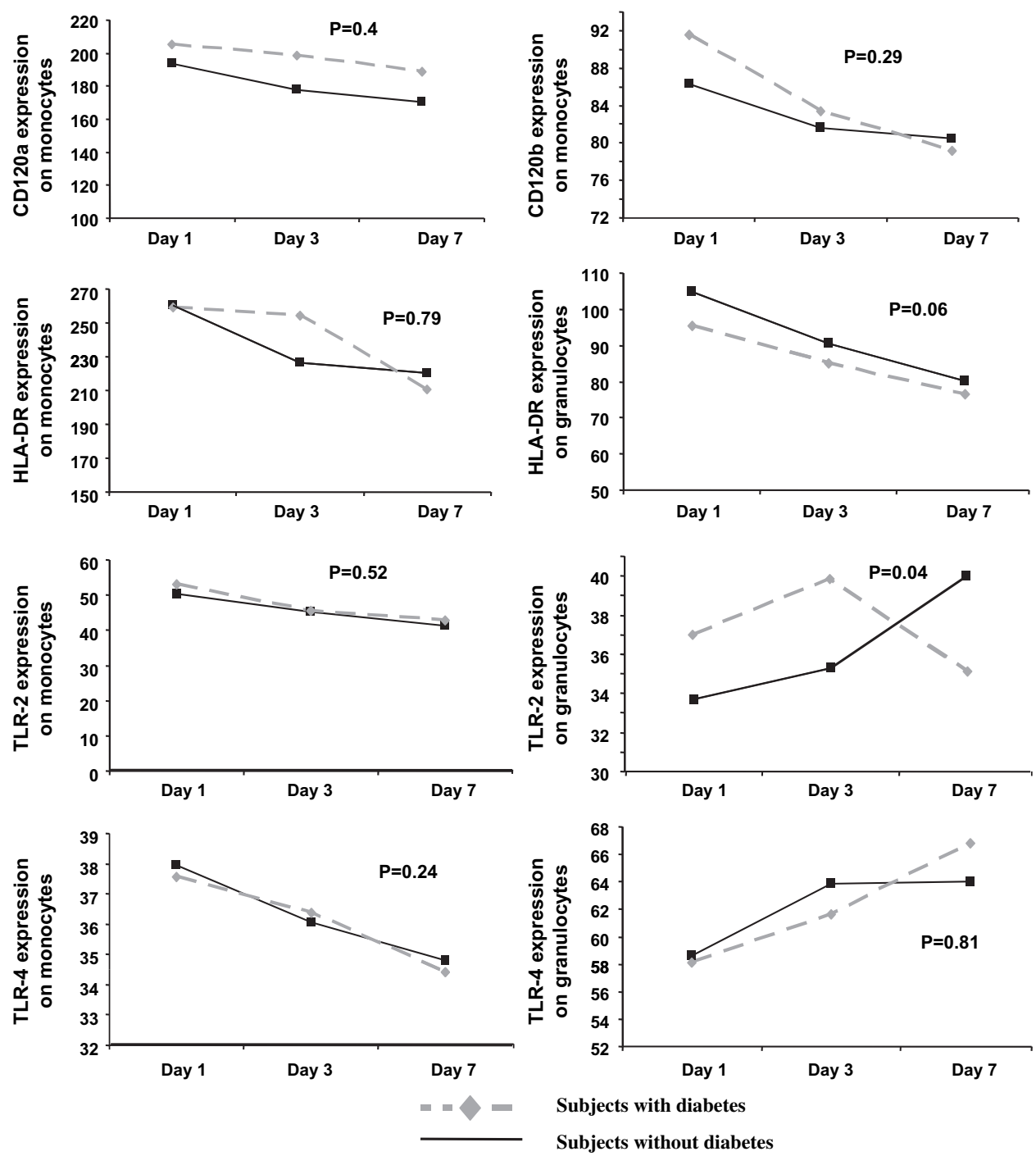
Table 5 Association between inflammatory, coagulation, fibrinolysis markers (geometric means with SD) at hospital discharge stratified by diabetes among 1808 subjects discharged alive from the hospital

\begin{tabular}{lrcl}
\hline Biomarker & With diabetes & Without diabetes & p Value \\
\hline Inflammatory markers $(\mathrm{pg} / \mathrm{ml})^{*}$ & & & \\
TNF & $4.77(2.12)$ & $4.73(2.65)$ & 0.51 \\
IL-6 & $8.67(6.83)$ & $7.41(5.74)$ & 0.03 \\
IL-10 & $1(18.45)$ & $1.24(11.92)$ & 0.6 \\
Coagulation markers* & & & \\
Factor IX activity (\%) & $111.02(1.30)$ & $119.14(1.25)$ & 0.1 \\
Antithrombin $(\mathrm{mg} / \mathrm{ml})$ & $91.77(1.04)$ & $91.50(1.04)$ & 0.87 \\
TAT complexes $(\mu \mathrm{g} / \mathrm{ml})$ & $4.25(3.70)$ & $3.58(2.49)$ & 0.25 \\
Fibrinolysis markers $(\mathrm{ng} / \mathrm{ml})^{*}$ & & & \\
D-dimer & $623.79(3.26)$ & $600.86(2.92)$ & 0.67 \\
PAl-1 & $3.54(4.27)$ & $3.72(4.63)$ & 0.62 \\
\hline
\end{tabular}

*Inflammatory, coagulation and fibrinolysis markers were available at hospital discharge in 1739,892 and 892 subjects, respectively.

IL, interleukin; PAl, plasminogen activator inhibitor; TAT, thrombin-antithrombin; TNF, tumour necrosis factor.

assessed survival differences after the first hospitalisation event in Health $\mathrm{ABC}$ to avoid confounding due to preceding noninfectious illnesses, such as acute myocardial infarction, which are common and may be associated with worse survival in diabetes. ${ }^{10}$ We also demonstrated an interaction between diabetes, reason for hospitalisation and mortality within the first year. Among those with diabetes, mortality was higher after CAP hospitalisation compared with hospitalisation for noninfectious illnesses. Thirdly, survival differences persisted after adjusting for differences in chronic disease burden, such as cardiovascular disease and CKD. These results suggest that the higher mortality within the first year in diabetes can be attributed to the CAP hospitalisation and cannot be explained by higher burden of pre-existing cardiovascular disease and CKD among those with diabetes.

We speculate that acceleration of pre-existing chronic disease may explain higher long-term mortality among those with diabetes. For instance, cardiovascular disease accounted for more than third of all deaths in individuals with diabetes. Pre-existing cardiovascular disease was more common among those with diabetes, which may be further accelerated by the acute infection. Early recognition or better management of atherosclerotic heart disease and concomitant risk factors, such as smoking and hyperlipidaemia, may improve outcomes. We showed that diabetes was associated with higher risk of acute kidney injury, which was associated with higher risk of 1-year mortality in our study and previous studies. ${ }^{30}$ Acute kidney injury itself or its sequela, CKD, may lead to death by several mechanisms, including increased risk of cardiovascular disease and infections.

Our results may explain the conflicting results of previous studies which assessed the association between diabetes and survival after CAP. First, mortality was assessed at different time points in earlier studies. Our results suggest that, although the RR of death was similar at different time points, statistically significant differences are more likely to be seen in studies that assessed long-term outcomes due to fewer deaths at earlier time points. ${ }^{3}$ Secondly, we examined the effect of diabetes on outcomes, conditional upon developing pneumonia. Previous population-based studies that showed higher incidence of death due to infections in subjects with diabetes could not assess whether these differences were due to increased susceptibility to infections or worse outcomes after infection. ${ }^{56}$

We showed a 1.3-fold higher risk of acute kidney injury among subjects with diabetes, even among subjects without a history of CKD. Mechanisms underlying increased risk of acute kidney injury after CAP among those with diabetes are unclear. We speculate that the higher risk of acute kidney injury in subjects with diabetes could be due to higher prevalence of subclinical kidney disease prior to pneumonia or due to higher risk of developing contrast-induced nephropathy. ${ }^{31} 32$

Although immune dysfunction in diabetes is well recognised, the lack of a clear influence of diabetes on a broad panel of biomarkers and cel surface markers at multiple time points during hospitalisation for CAP is remarkable. We observed differences in expression of TLR2 on granulocytes over time between subjects with and without diabetes, but the direction of this difference was not consistent over time and was uncertain in terms of biological significance. We chose inflammatory, coagulation and fibrinolysis biomarkers because these biomarkers are altered in diabetes ${ }^{1133}$ and in human endotoxaemia models by hyperinsulinaemia and hyperglycaemia. ${ }^{12} 14$ We chose TLR2 and TLR4 because expression of these cell surface markers and their downstream mediators is upregulated in diabetes ${ }^{16}$ and they play an important role in host response to infection and sepsis. ${ }^{15}$ We performed serial measurements of these markers, including on presentation to the ED, when immune responses are least likely to be modified by therapeutic interventions. It is likely that diabetes may modify these markers prior to ED presentation, or other mechanisms could be influenced by diabetes, such as alterations in neutrophil function, apoptosis, oxidative stress and chemokines. However, the lack of effect of diabetes on the immune response in a broad panel of circulating biomarkers and cell surface markers suggest that pre-existing diabetes does not influence the immediate host response to CAP, possibly because the responses elicited by pneumonia are much more profound than the relatively modest alterations produced by diabetes per se.

Our study has limitations. First, we assessed the effect of hyperglycaemia, a potential mechanism of increased mortality, on ED presentation only, and serial glucose levels were not available. Secondly, we used different criteria to diagnose diabetes in both cohorts. Stringent criteria were used in Health $\mathrm{ABC}$, including self-report and review of medication inventory, and fasting blood glucose in subjects who did not report diabetes. In GenIMS, we used a combination of self-report and review of medication inventory, and we may have misclassified some individuals as non-diabetic. The association between admission hyperglycaemia and higher mortality only among those without diabetes in this cohort suggests that such a misclassification bias would skew the results towards the null and attenuate the HRs between diabetes and long-term survival. Indeed, the HRs were slightly lower in GenIMS, suggesting that such a misclassification bias is unlikely to negate our results. Finally, we could not assess whether our results were confounded due to differences in microbiological aetiology between those with and without diabetes. Although cultures were obtained in most subjects, we identified an aetiological agent in a small subgroup. The low yield of cultures in our study is consistent with previous large studies of patients with $\mathrm{CAP}^{3435}$ and probably due to poor yield of current culture techniques. Larger studies will be necessary to understand differences in immune response for different aetiological agents.

In summary, once CAP occurs, those with diabetes were more likely to die over 1 year. The mechanism is unlikely to be due to alterations in immune response, at least as measured by a broad panel of circulating biomarkers and cell surface markers. The higher mortality may be due to worsening of pre-existing cardiovascular disease or higher risk of acute kidney injury. 
Acknowledgements We are indebted to the nurses, respiratory therapists, phlebotomists, physicians and other healthcare professionals who participated in GenIMS, as well as the subjects and their families who supported GenIMS and the Health $A B C$ study. A complete list of GenlMS investigators is available at http://www.ccm.upmc.edu/genims investigators

Funding GenIMS was funded by NIGMS R01 GM61992 with additional support from GlaxoSmithKline for enrolment and clinical data collection, and Diagnostic Products Corporation for the cytokine assays. Health ABC was funded by NIA (N01-AG-6-2101, N01-AG-6-2103, N01-AG-6-2106), NHLBI (R01HL74104) and NIAID (27913 and 39482). Health $A B C$ was supported in part by the Intramural Research Program of the $\mathrm{NIH}$, National Institute on Ageing. SY is supported by K23GM083215.

Competing interests None.

Ethics approval This study was conducted with the approval of the ethics committee at each participating site.

Provenance and peer review Not commissioned; externally peer reviewed.

\section{REFERENCES}

1. Shah BR, Hux JE. Quantifying the risk of infectious diseases for people with diabetes. Diabetes Care 2003;26:510-13.

2. Muller LM, Gorter KJ, Hak E, et al. Increased risk of common infections in patients with type 1 and type 2 diabetes mellitus. Clin Infect Dis 2005;41:281-8.

3. Falguera $\mathbf{M}$, Pifarre $\mathrm{R}$, Martin $\mathrm{A}$, et al. Etiology and outcome of community-acquired pneumonia in patients with diabetes mellitus. Chest 2005;128:3233-9.

4. Kornum JB, Thomsen RW, Riis A, et al. Type 2 diabetes and pneumonia outcomes: a population-based cohort study. Diabetes Care 2007;30:2251-7.

5. Benfield T, Jensen JS, Nordestgaard BG. Influence of diabetes and hyperglycaemia on infectious disease hospitalisation and outcome. Diabetologia 2007;50:549-54.

6. Valdez R, Narayan KM, Geiss LS, et al. Impact of diabetes mellitus on mortality associated with pneumonia and influenza among non-Hispanic black and white US adults. Am J Public Health 1999;89:1715-21.

7. Esper A, Moss M, Martin G. The effect of diabetes mellitus on organ dysfunction with sepsis: an epidemiological study. Crit Care 2009;13:R18.

8. US Renal Data Systems. USRDS 2006 Annual Data Report: Atlas of End-stage Renal Disease in the United States. Bethesda, MD: National Institutes of Health National Institute of Diabetes and Digestive and Kidney Diseases, 2007. http://www.usrds.org (accessed Dec 2008)

9. Centers for Disease Control and Prevention (CDC). Prevalence of self-reported cardiovascular disease among persons aged $>35$ years with diabetes-United States, 1997-2005. MMWR Morb Mortal Wkly Rep 2007:56:1129-32.

10. Timmer JR, Ottervanger JP, Thomas K, et al. Long-term, cause-specific mortality after myocardial infarction in diabetes. Eur Heart J 2004;25:926-31.

11. Lu H, Raptis M, Black E, et al. Influence of diabetes on the exacerbation of an inflammatory response in cardiovascular tissue. Endocrinology 2004;145:4934-9.

12. Krogh-Madsen R, Moller K, Dela F, et al. Effect of hyperglycemia and hyperinsulinemia on the response of IL-6, TNF-\{alpha\}, and FFAs to low-dose endotoxemia in humans. Am J Physiol Endocrinol Metab 2004;286:E766-72.

13. Reverter JL, Reverter JC, Tassies D, et al. Thrombomodulin and induced tissue factor expression on monocytes as markers of diabetic microangiopathy: a prospective study on hemostasis and lipoproteins in insulin-dependent diabetes mellitus. Am J Hematol 1997:56:93-9.

14. Stegenga ME, van der Crabben SN, Blumer RME, et al. Hyperglycemia enhances coagulation and reduces neutrophil degranulation, whereas hyperinsulinemia inhibits fibrinolysis during human endotoxemia. Blood 2008:112:82-9.

15. Cohen J. The immunopathogenesis of sepsis. Nature 2002;420:885-91.

16. Devaraj S, Dasu MR, Rockwood J, et al. Increased Toll-Like Receptor (TLR) 2 and TLR4 expression in monocytes from patients with Type 1 diabetes: further evidence of a proinflammatory state. J Clin Endocrinol Metab 2008;93:578-83.

17. Levy MM, Fink MP, Marshall JC, et al. 2001 SCCM/ESICM/ACCP/ATS/SIS International Sepsis Definitions Conference. Crit Care Med 2003:31:1250-6.

18. Barzilay JI, Spiekerman CF, Wahl PW, et al. Cardiovascular disease in older adults with glucose disorders: comparison of American Diabetes Association criteria for diabetes mellitus with WHO criteria. Lancet 1999;354:622-5.

19. Levey AS, Bosch JP, Lewis JB, et al. A more accurate method to estimate glomerular filtration rate from serum creatinine: a new prediction equation. Modification of Diet in Renal Disease Study Group. Ann Intern Med 1999;130:461-70.

20. Yende S, Angus DC, Ali IS, et al. Influence of comorbid conditions on long-term mortality after pneumonia in older people. J Am Geriatr Soc 2007:55:518-25.

21. National Death Index. http://www.cdc.gov/nchs/ndi.htm (accessed Dec 2009).

22. Cowper DC, Kubal JD, Maynard C, et al. A primer and comparative review of major U.S. mortality databases. Ann Epidemiol 2002;12:462-8.
23. Bellomo R, Ronco C, Kellum JA, et al. Acute renal failure-definition, outcome measures, animal models, fluid therapy and information technology needs: the Second International Consensus Conference of the Acute Dialysis Quality Initiative (ADOI) Group. Crit Care 2004:8:R204-12.

24. Knaus WA, Wagner DP, Draper EA, et al. The APACHE III prognostic system. Risk prediction of hospital mortality for critically ill hospitalized adults. Chest 1991;100:1619-36.

25. Fine MJ, Auble TE, Yealy DM, et al. A prediction rule to identify low-risk patients with community acquired pneumonia. N Engl J Med 1997;336:243-50.

26. Kellum JA, Kong L, Fink MP, et al. Understanding the inflammatory cytokine response in pneumonia and sepsis: results of the Genetic and Inflammatory Markers of Sepsis (GenIMS) Study. Arch Intern Med 2007:167:1655-63.

27. Yende S, D'Angelo G, Kellum JA, et al. Inflammatory markers at hospital discharge predict subsequent mortality after pneumonia and sepsis. Am J Respir Crit Care Med 2008:177:1242-7.

28. Epstein MP, Lin X, Boehnke M. A tobit variance-component method for linkage analysis of censored trait data. Am J Hum Genet 2003;72:611-20.

29. Hochberg Y, Benjamini Y. More powerful procedures for multiple significance testing. Stat Med 1990;9:811-18.

30. Abosaif NY, Tolba YA, Heap M, et al. The outcome of acute renal failure in the intensive care unit according to RIFLE: model application, sensitivity, and predictability. AmJ Kidney Dis 2005;46:1038-48.

31. Mehran R, Aymong ED, Nikolsky $\mathrm{E}$, et al. A simple risk score for prediction of contrast-induced nephropathy after percutaneous coronary intervention: development and initial validation. J Am Coll Cardiol 2004;44:1393-9.

32. Ismail N, Becker B, Strzelczyk $P$, et al. Renal disease and hypertension in non-insulin-dependent diabetes mellitus. Kidney Int 1999;55:1-28.

33. Graves DT, Liu R, Alikhani $\mathrm{M}$, et al. Diabetes-enhanced inflammation and apoptosis - impact on periodontal pathology. J Dent Res 2006;85:15-21.

34. Metersky ML, Ma A, Bratzler DW, et al. Predicting bacteremia in patients with community-acquired pneumonia. Am J Respir Crit Care Med 2004;169:342-7.

35. Joshi N, Caputo GM, Weitekamp MR, et al. Infections in patients with diabetes mellitus. N Engl J Med 1999:341:1906-12.

\section{APPENDIX 1: LIST OF NON-INFECTIOUS CAUSES OF HOSPITALISATION IN HEALTH ABC STUDY}

Myocardial infarction

Angina/other ischaemic disease

Congestive heart failure

Carotid artery disease

Peripheral arterial disease

Stroke or cerebrovascular accident (CVA)

Transient ischaemic attack

Chronic obstructive pulmonary disease/emphysema/asthma

Upper gastrointestinal bleeding

Lower gastrointestinal bleeding

Abdominal hernia

Benign prostatic hyperplasia

Gallbladder disease

Cancer

Depression

Dementia

Osteoarthritis

Fracture

Neoplasms

Endocrine, nutrition, metabolic diseases

Diseases of blood and blood-forming organs

Mental disorders (not dementia and depression)

Diseases of nervous system, other than stroke

Diseases of circulatory system, other than myocardial infarction, angina and congestive heart failure

Diseases of respiratory system, other than chronic obstructive pulmonary disease asthma, emphysema

Diseases of digestive system, other than bleeding

Diseases of genitourinary system, other than benign prostatic hyperplasia

Diseases of skin

Diseases of musculoskeletal and connective tissue, other than osteoarthritis

III-defined symptoms and signs

Injury and poisoning, excluding fractures 